\title{
A Data-Adaptive Compressed Sensing Approach to Polarimetric SAR Tomography of Forested Areas
}

\author{
Esteban Aguilera, Matteo Nannini, and Andreas Reigber, Senior Member, IEEE
}

\begin{abstract}
Super-resolution imaging via compressed sensing (CS)-based spectral estimators has been recently introduced to synthetic aperture radar (SAR) tomography. In the case of partial scatterers, the mainstream has so far been twofold, in that the tomographic reconstruction is conducted by either directly working with multiple looks and/or polarimetric channels or by exploiting the corresponding single-channel second-order statistics. In this letter, we unify these two methodologies in the context of covariance fitting. In essence, we exploit the fact that both vertical structures and the unknown polarimetric signatures can be approximated in a low-dimensional subspace. For this purpose, we make use of a wavelet basis in order to sparsely represent vertical structures. Additionally, we synthesize a data-adaptive orthonormal basis that spans the space of polarimetric signatures. Finally, we validate this approach by using fully polarimetric L-band data acquired by the E-SAR sensor of the German Aerospace Center (DLR).
\end{abstract}

Index Terms-Distributed compressed sensing (DCS), Kronecker basis, polarimetry, synthetic aperture radar (SAR) tomography, wavelets.

\section{INTRODUCTION}

$\mathbf{M}$ ULTIBASELINE polarimetric measurements allow us to resolve a vertical structure via well-established synthetic aperture radar (SAR) imaging principles. Also, they provide an additional dimension to further describe the response of illuminated objects, which, in the case of partial scatterers, is commonly captured in the form of a polarimetric covariance matrix. However, the achievable resolution of conventional estimators is highly dependent on the extension of the elevation aperture (see Fig. 1). Moreover, the sampling rate dictated by the well-known Nyquist frequency imposes an additional requirement, namely, dense regular sampling [1].

Recently, alternative sparsity-driven nonlinear reconstruction algorithms have been put forward in order to attain low sidelobe and ambiguity levels with a reduced number of irregular passes. In particular, the authors in [2] extended the work in [3] and [4] and proposed a compressed sensing (CS)based joint reconstruction technique that takes advantage of possible intersignal structural correlations between neighboring azimuth-range pixels, as well as between polarimetric channels. Also, a single-channel covariance fitting methodology was introduced in [5], which employs sparse representations of the vertical backscattered power in the wavelet domain. In this

Manuscript received March 22, 2012; revised June 13, 2012 and July 20, 2012; accepted August 6, 2012.

The authors are with the Microwaves and Radar Institute (HR), German Aerospace Center (DLR), 82234 Wessling, Germany (e-mail: Esteban.Aguilera@dlr.de).

Digital Object Identifier 10.1109/LGRS.2012.2212693

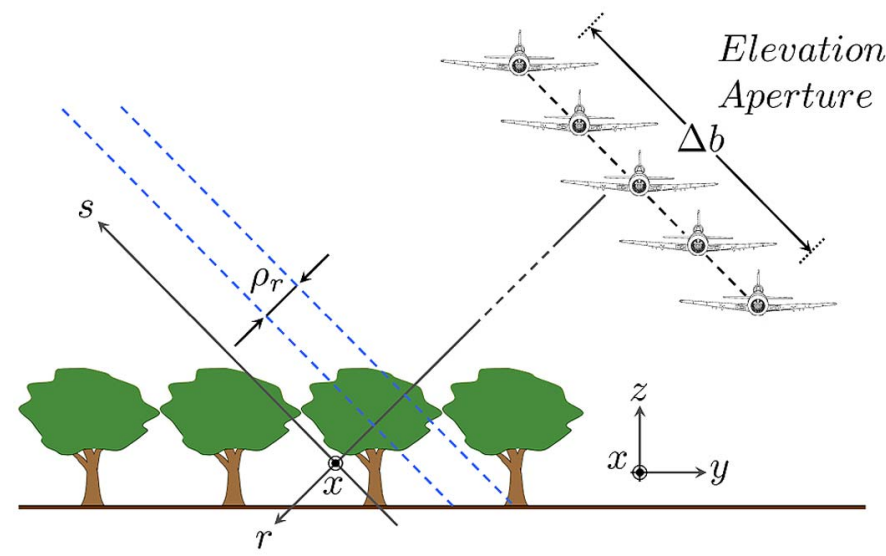

Fig. 1. Tomographic sensing operation using parallel passes (not to scale). The range resolution is indicated by $\rho_{r}$, whereas the extension of the elevation aperture by $\Delta b$.

letter, we combine all these lines of research and extend them so as to take advantage of the fact that polarimetric signatures, as characterized by the so-called coherency/covariance matrix (from now on referred to as polarimetric covariance matrix) [6], can be approximated in a low-dimensional subspace [7]. For this purpose, we form a data-adaptive orthonormal basis that spans the space of polarimetric signatures.

The remainder of the letter is organized as follows. In Section II, we formulate the inverse problem from a multibaseline polarimetric covariance matrix perspective in such a way that we are able to decouple the structures of scattering mechanisms (SMs) from their polarimetric signatures. Subsequently, Section III revisits the concept of joint sparse reconstruction. Section IV casts the covariance fitting problem as an instance thereof and reformulates it so as to be able to restrict the polarimetric signatures to lie in a low-dimensional subspace. In Section V, we present results obtained using fully polarimetric L-band data acquired by one of the airborne sensors of the German Aerospace Center (DLR), namely, E-SAR. Finally, Section VI concludes this letter.

\section{Problem Formulation}

Let $K_{i, j} \in \mathbb{C}^{m \times m}$ be the multibaseline covariance matrix resulting from $m$ parallel passes [1], [8] and two polarimetric channels $i$ and $j$ at a specific azimuth-range position, with $1 \leq i, j \leq 3$. For example, $i$ and $j$ could denote the $h h$ and $h v$ channels, respectively. Then, we can construct $D \in \mathbb{C}^{m^{2} \times 9}$ as follows:

$$
D=\left[\operatorname{vec}\left(K_{1,1}\right) \quad \operatorname{vec}\left(K_{2,1}\right) \quad \cdots \quad \operatorname{vec}\left(K_{3,3}\right)\right]
$$


where $\operatorname{vec}(\cdot)$ is the matrix-to-vector operator. Additionally, under suitable assumptions [5], [7], [9], $D$ can be written out as a sum of contributions of $S$ SMs, i.e.,

$$
D=\Phi \sum_{s=1}^{S} \theta_{s} C_{s}^{T}=\Phi Z
$$

where $\Phi \in \mathbb{C}^{m^{2} \times n}$ is a partial Fourier matrix, $\theta_{s} \in \mathbb{R}_{>0}^{n}$ accounts for the vertical power distribution of the $s$ th SM (i.e., its vertical structure is described by $n$ pixels), $C_{s} \in \mathbb{C}^{9}$ refers to the polarimetric signature of the sth SM (which can be rearranged into a three-by-three polarimetric covariance matrix), and hence $Z \in \mathbb{C}^{n \times 9}$. Accordingly, we will focus on reconstructing $Z$, albeit always driven by the structure conveyed by $\theta_{s}$ and $C_{s}$.

Finally, we point out that, by construction, the number of independent measurements provided by the entries of $D$ amounts, at most, to $3 m(3 m+1) / 2$ (rather than $9 m^{2}$ ) and can be optimized by means of a minimum redundancy acquisition geometry [5], [10]. Note also that, although (2) clearly neglects any source of decorrelation, this aspect can be explicitly incorporated as a part of the tomographic reconstruction (see Section IV).

\section{Multisignal CS}

Multisignal CS enables the joint recovery of signal ensembles by exploiting intersignal structural correlations. It generalizes the concept of a signal being sparse to the concept of an ensemble of signals being jointly sparse [11], [12]. In particular, it proposes taking linear measurements of the form $B=A X+Y$, where $X \in \mathbb{C}^{n \times L}$ indicates $L$ sparse signals of interest that exhibit common support, $A \in \mathbb{C}^{m \times n}$ is a sensing matrix with $m$ usually much smaller than $n$, and $Y \in \mathbb{C}^{m \times L}$ is an unknown perturbation term. The theory asserts that, under suitable conditions [2], [11], [13], $X$ can be recovered by mixed norm minimization, i.e.,

$$
\min _{\widetilde{X}}\|\widetilde{X}\|_{2,1} \text { subject to }\|A \widetilde{X}-B\|_{F} \leq \varepsilon
$$

where $\varepsilon$ is an upper bound on the perturbation level, $\|\cdot\|_{F}$ is the Frobenius matrix norm, and $\|\cdot\|_{2,1}$ is a mixed norm (sum of the $L_{2}$ norms of the rows of a matrix) that basically promotes sparsity along columns while minimizing the energy along rows. As a consequence, joint reconstruction guarantees recovery, even when $m<n$, by promoting row sparsity.

\section{Covariance Fitting via Multisignal CS}

In this section, we formulate the reconstruction of $Z$ as defined in (2) from a multisignal CS perspective. To that end, we first observe that forested areas are generally dominated by few effective SMs. Specifically, the assumption of simply two SMs has recently proven to be a valid approximation [7], [9], [14]. In addition, the power distribution $\theta_{s}$ of these different SMs is quite regular [7], [8], [14], [15], thereby giving rise to sparse representations in the wavelet domain and allowing for a CS viewpoint [5]. Consequently, if we let $W \in \mathbb{R}^{n \times n}$ be a sparsifying basis for $\theta_{s}$, with $s \leq 2$, it follows that $Z$ can be represented by a row-sparse matrix $\alpha \in \mathbb{C}^{n \times 9}$, such that $Z=W^{T} \alpha$. Incidentally, the condition $s \leq 2$ can be readily satisfied by replacing $D$ with its best rank-2 approximation, which will be denoted by $D_{2}$. As thoroughly discussed in [7], this can be efficiently obtained by means of a singular-value decomposition (SVD).

\section{A. Naive Approach}

As a stepping-stone toward a robust formulation, we can simply take (2) and (3), and let $B=D_{2}, A=\Phi W^{T}$, and $X=\alpha$. Then, we could recover $\alpha$ by carrying out the following optimization:

$$
\min _{\widetilde{\alpha}}\|\widetilde{\alpha}\|_{2,1} \text { subject to }\left\|\Phi W^{T} \widetilde{\alpha}-D_{2}\right\|_{F} \leq \varepsilon
$$

where $\varepsilon$ is an upper bound on the model mismatch that potentially captures any source of decorrelation and insufficient level of multilooking [5]. In turn, we would compute $\widetilde{Z}=W^{T} \widetilde{\alpha}$. Although intuitive and simple, this approach falls short of ideal, in that it ignores the inherent properties and low dimensionality of the subspace of polarimetric signatures, which is the subject of Section IV-B.

\section{B. Data-Adaptive Approach}

As previously mentioned, the SVD of $D$ provides an efficient way of computing its best rank-2 approximation. Nonetheless, of equal importance is the fact that the adjoint of the corresponding right singular vectors defines a data-adaptive orthonormal basis for the unknown polarimetric signatures [7] (see also [16] and [17]). As a result, if we consider only two SMs, i.e., $D_{2}$, it follows that every row of $Z$ will be bound to lie in a 2-D subspace. Thus, once two orthonormal vectors, i.e., $v_{1}$ and $v_{2} \in \mathbb{C}^{9}$, that span the polarimetric space have been obtained and a matrix $V \in \mathbb{C}^{2 \times 9}$, i.e.,

$$
V=\left[\begin{array}{l}
v_{1}^{T} \\
v_{2}^{T}
\end{array}\right]
$$

has been formed, we can formulate a data-adaptive reconstruction as

$$
\min _{\widetilde{\beta}}\|\widetilde{\beta}\|_{2,1} \text { subject to }\left\|\Phi W^{T} \widetilde{\beta} V-D_{2}\right\|_{F} \leq \varepsilon
$$

where $\beta \in \mathbb{R}^{n \times 2}$. Just as in Section IV-A, $\varepsilon$ is an upper bound on the model mismatch. Then, we have $\widetilde{Z}=W^{T} \widetilde{\beta} V$. In order to ensure physical validity, proper constraints must be set so that every row of $\widetilde{Z}$ (when rearranged in a three-by-three matrix) results in a positive-semidefinite matrix. To this end, let $V_{i} \in$ $\mathbb{C}^{3 \times 3}$ and $\widetilde{Z}_{j} \in \mathbb{C}^{3 \times 3}$ be the result of rearranging $v_{i}$ and $\widetilde{z}_{j} \in$ $\mathbb{C}^{9}$ (a vector corresponding to the $j$ th row of $\widetilde{Z}$ ) into three-bythree matrices, respectively. Since it is possible to find a matrix $G$ that jointly diagonalizes $V_{1}$ and $V_{2}$ (see, for example, [7]), it follows that $G^{*} \widetilde{Z}_{j} G$ is certain to be diagonal. Furthermore, the corresponding main diagonal entries can be alternatively computed by $M \widetilde{z}_{j}$, where $M \in \mathbb{C}^{3 \times 9}$ is formed from $G^{*}$ and $G$. Then, the required positive semidefiniteness can be enforced by adding a constraint of the form $M\left(W^{T} \widetilde{\beta} V\right)^{T} \succeq 0$. 


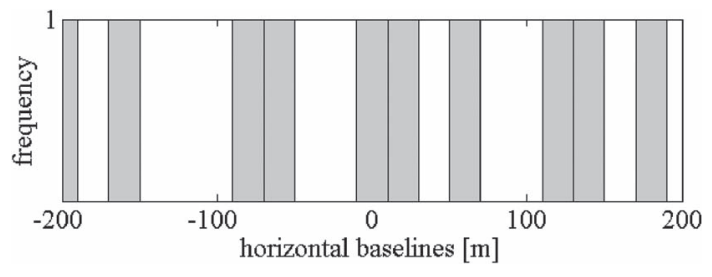

Fig. 2. Histogram of the baseline distribution corresponding to ten irregular parallel passes.

Interestingly, this approach can be understood from a slightly different, yet instructive, viewpoint. Specifically, rather than considering $Z$ to be composed of nine 1-D signals (column vectors), we can think of it as one 2-D signal. As a result, $Z$ can be represented in a 2-D basis, readily formed by computing the outer product of all 1-D wavelet basis vectors and all 1-D polarimetric signature basis vectors, i.e., a Kronecker basis. Thus, just as the former allows for sparse expansions of the vertical backscattered power, the latter provides a sparse expansion of the polarimetric signature. However, since we only consider two SMs, the support of the polarimetric signature transform coefficients is known a priori. Consequently, if we let $\ell \ll n$ be the size of the union of the supports of the two vertical power distributions (in the wavelet domain), then the number of nonzero (real-valued) transform coefficients reduces to $2 \ell$. We emphasize that this dimensionality holds for dual-polarized and fully polarimetric acquisitions since, in both cases, the polarimetric signatures are bound to lie in a 2-D subspace. Finally, we note that, from this perspective, $\Phi$ is bound to take partitioned measurements, in that only one column is measured at a time. For further details on this kind of distributed sensing setting, we refer the reader to [18].

\section{EXPERIMENTAL RESULTS}

For validation purposes, we used a stack of ten focused and coregistered SAR images obtained by processing fully polarimetric L-band data. These data were acquired by the E-SAR airborne sensor of DLR during a campaign near Dornstetten, Germany, in 2006. Fig. 2 shows the histogram of the corresponding irregular baseline distribution. Fig. 3 shows the amplitude image of this area. The center frequency used was $1.3 \mathrm{GHz}$, and the nominal altitude above ground was about $3200 \mathrm{~m}$. The resolutions were 0.66 and $2.07 \mathrm{~m}$ in azimuth and range, respectively [8].

The tomographic reconstruction was conducted as follows. We recast (6) as

$$
\min _{\widetilde{\beta}} \lambda\|\widetilde{\beta}\|_{2,1}+\left\|\Phi W^{T} \widetilde{\beta} V-D_{2}\right\|_{F}^{2}
$$

with appropriate physical constraints, as developed in Section IV-B. Thus, in this formulation, $\lambda$ trades row sparsity for data mismatch. The sparsifying basis $W \in \mathbb{R}^{128 \times 128}$ was based on the Daubechies Symmlet wavelet with four vanishing moments and three levels of decomposition. Next, we selected several azimuth positions at a fixed range distance of $4816.30 \mathrm{~m}$, as indicated by the yellow rectangle in Fig. 3. In order for (2) to hold, we computed the sample covariance matrix

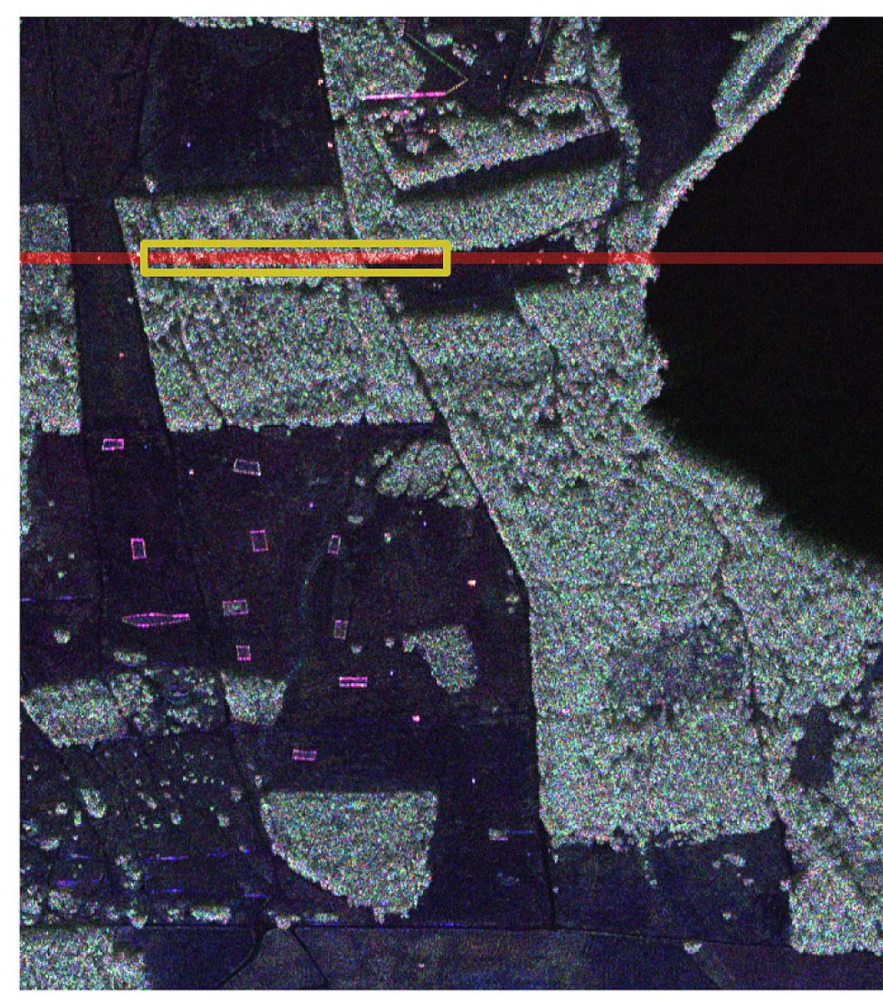

Fig. 3. Polarimetric SAR image of the test site near Dornstetten, Germany (red: $|h h-v v| / \sqrt{2}$; green: $\sqrt{2}|h v|$; blue: $|h h+v v| / \sqrt{2}$ ). The targets of interest are located within the yellow rectangle along azimuth.

by taking a $20 \times 20 \mathrm{~m}^{2}$ estimation window. In this respect, we employed the Sum of Kronecker Products (SKP) decomposition [7] using different window sizes in order to heuristically find the smallest window that allowed for the separation of SMs. Since the value of $\lambda$ is not known a priori, we proceeded heuristically as well. First, we performed the reconstruction (in the Lexicographic basis) repeatedly with increasing $\lambda$ starting from 0 (i.e., no regularization). We then chose the smallest value such that all the recovered profiles exhibited no spikes, so as to be consistent with the assumption that the unknown vertical profiles are sparse in the wavelet domain. In this regard, we note that spikes or rather spatially sparse solutions are expected in this kind of optimization when it is not regularized but nonnegativity constraints are imposed (see, for example, [19]). For this specific tomographic slice, we decided upon $\lambda=2$. As a result, we obtained slices in the azimuth and elevation directions of dimensions $300 \mathrm{~m}$ by $40 \mathrm{~m}$, respectively. Figs. 4-6 show a comparison of the reconstructed profiles for the $h h, v v$, and $h v$ channels, respectively. The tomograms found by means of the outlined methodology are shown first, followed by a conventional linear inversion of the $K_{i, j}$ matrices previously defined but considering only two SMs. As conveyed by the figures, the CS reconstruction is able to counter the artifacts exhibited by the linear reconstruction, which originate from the irregular baselines. Additionally, Fig. 7 shows the correlations between the $v v$ and $h h$ channels (as taken from $\widetilde{Z}$ ), which clearly manifest more strongly at the ground level. In order to estimate the effective dimensionality of $\widetilde{Z}$, we computed $W \widetilde{Z}$ and sorted its rows according to their $L_{2}$ norms. Then, 


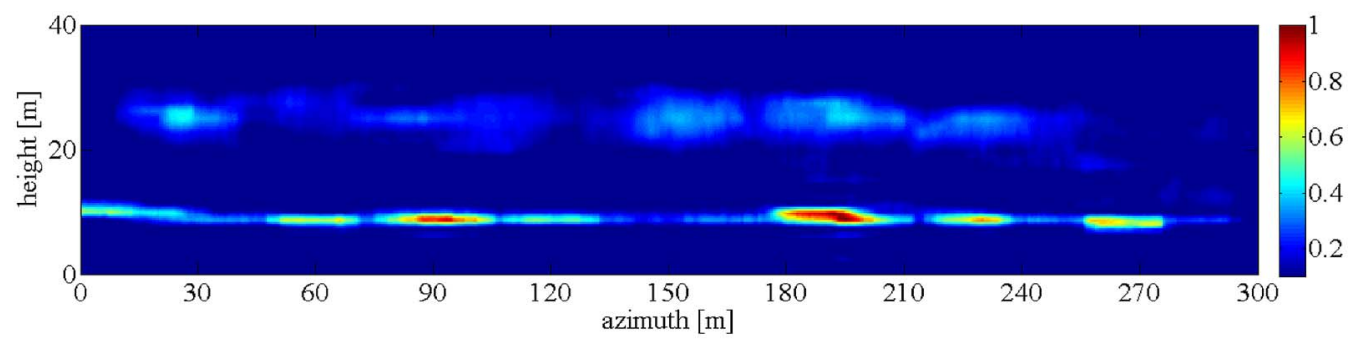

(a)

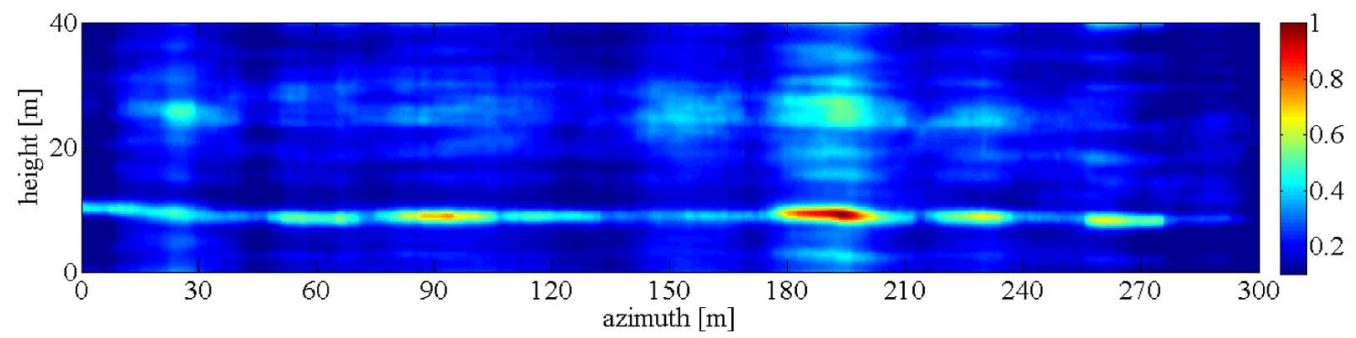

(b)

Fig. 4. Tomographic slices in the $h h$ channel ( $300 \mathrm{~m}$ by $40 \mathrm{~m}$ ) using a $20 \times 20 \mathrm{~m}^{2}$ window (normalized to the maximum value of the tomogram), obtained (a) by means of the data-adaptive CS approach and (b) by a conventional linear inversion.

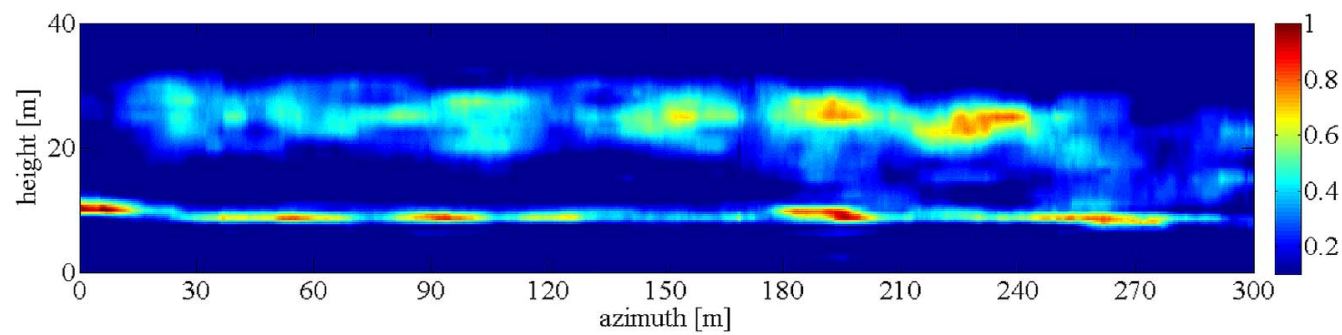

(a)

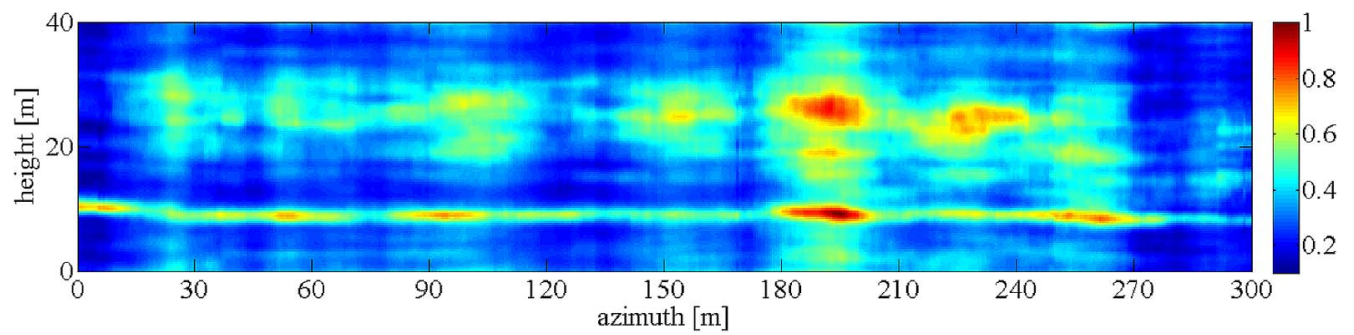

(b)

Fig. 5. Tomographic slices in the $v v$ channel $\left(300 \mathrm{~m}\right.$ by $40 \mathrm{~m}$ ) using a $20 \times 20 \mathrm{~m}^{2}$ window (normalized to the maximum value of the tomogram), obtained (a) by means of the data-adaptive CS approach and (b) by a conventional linear inversion.

we observed that, for every azimuth position, the largest seven rows (out of 128) retain at least $91 \%$ of the energy, whereby we conclude that $\ell \approx 7$.

The solver that we used was CVX, which is a package for specifying and solving convex programs [20]. For large-scale processing, we refer the reader to [14] for a detailed discussion on how to reduce the size of this kind of problem.

\section{CONCLUSION}

In this letter, we have extended the existing CS-based methodologies by considering the multiple dimensions in which polarimetric covariance matrices, i.e., polarimetric signatures, manifest. Thus, we have been able to perform joint recovery by exploiting the fact that vertical structures, as well as polarimetric signatures, can be sparsely approximated in appropriate domains. For this purpose, we have made use of a wavelet basis and a data-adaptive basis, respectively, thereby reducing the degrees of freedom to carry out the tomographic reconstruction. In this regard, we emphasize that the outlined approach is not limited to wavelets as, in fact, any suitable basis could be used. Also, we stress the fact that the implementation of this technique can be effectively tailored by means of existing tools, such as the SKP decomposition. Future publications will focus on analyzing the robustness of the proposed approach by considering different range distances and multibaseline constellations, and by comparing it with other traditional spectral estimators. 


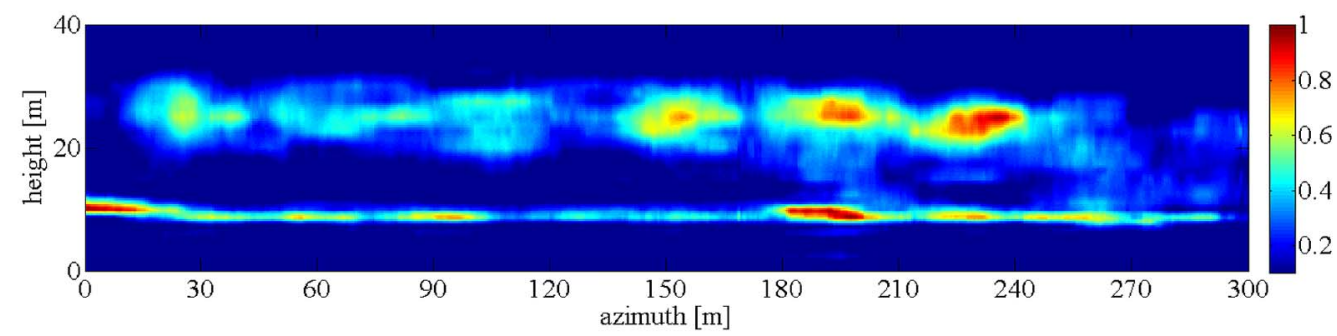

(a)

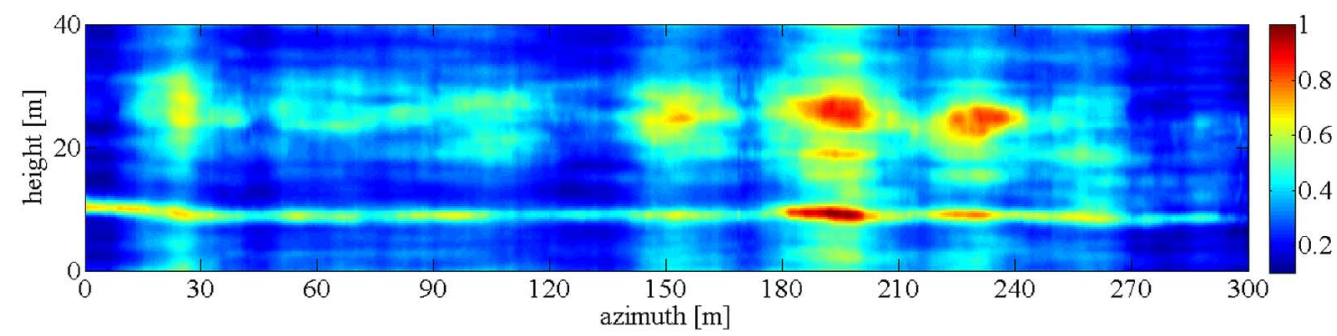

(b)

Fig. 6. Tomographic slices in the $h v$ channel ( $300 \mathrm{~m}$ by $40 \mathrm{~m}$ ) using a $20 \times 20 \mathrm{~m}^{2}$ window (normalized to the maximum value of the tomogram), obtained (a) by means of the data-adaptive CS approach and (b) by a conventional linear inversion.

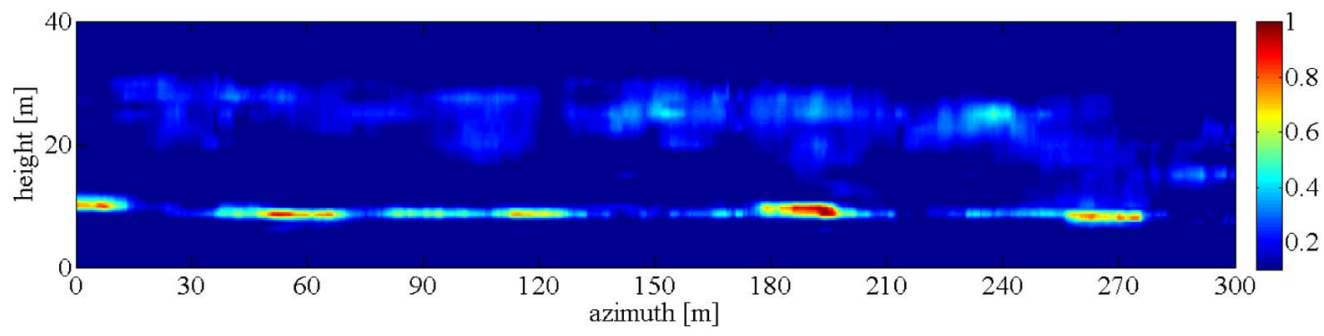

Fig. 7. Correlations between the $v v$ and $h h$ channels obtained by means of the data-adaptive CS approach $(300 \mathrm{~m}$ by $40 \mathrm{~m})$ using a $20 \times 20 \mathrm{~m}^{2}$ window (normalized to the maximum value of the tomogram).

\section{ACKNOWLEDGMENT}

The authors would like to thank Dr. S. Tebaldini for providing clear, concise, and valuable suggestions for the implementation of the SKP decomposition.

\section{REFERENCES}

[1] A. Reigber and A. Moreira, "First demonstration of airborne SAR tomography using multibaseline L-band data," IEEE Trans. Geosci. Remote Sens., vol. 38, no. 5, pp. 2142-2152, Sep. 2000.

[2] E. Aguilera, M. Nannini, and A. Reigber, "Multisignal compressed sensing for polarimetric SAR tomography," IEEE Geosci. Remote Sens. Lett., vol. 9, no. 5, pp. 871-875, Sep. 2012.

[3] X. Zhu and R. Bamler, "Tomographic SAR inversion by L1-norm regularization-The compressive sensing approach," IEEE Trans. Geosci. Remote Sens., vol. 48, no. 10, pp. 3839-3846, Oct. 2010.

[4] A. Budillon, A. Evangelista, and G. Schirinzi, "Three-dimensional SAR focusing from multipass signals using compressive sampling," IEEE Trans. Geosci. Remote Sens., vol. 49, no. 1, pp. 488-499, Jan. 2011.

[5] E. Aguilera, M. Nannini, and A. Reigber, "Wavelet-based compressed sensing for SAR tomography of forested areas," in Proc. EUSAR, 2012, pp. 259-262.

[6] S. Cloude and E. Pottier, "A review of target decomposition theorems in radar polarimetry," IEEE Trans. Geosci. Remote Sens., vol. 34, no. 2, pp. 498-518, Mar. 1996.

[7] S. Tebaldini, "Algebraic synthesis of forest scenarios from multibaseline PolInSAR data," IEEE Trans. Geosci. Remote Sens., vol. 47, no. 12, pp. 4132-4142, Dec. 2009.

[8] M. Nannini, R. Scheiber, R. Horn, and A. Moreira, "First 3-D reconstructions of targets hidden beneath foliage by means of polarimetric SAR tomography," IEEE Geosci. Remote Sens. Lett., vol. 9, no. 1, pp. 60-64, Jan. 2011.
[9] S. Tebaldini and F. Rocca, "Multibaseline polarimetric SAR tomography of a boreal forest at P- and L-bands," IEEE Trans. Geosci. Remote Sens., vol. 50, no. 1, pp. 232-246, Jan. 2012.

[10] M. Nannini, A. Reigber, and R. Scheiber, "A study on irregular baseline constellations in SAR tomography," in Proc. EUSAR, 2010, pp. 1-4.

[11] Y. Eldar and H. Rauhut, "Average case analysis of multichannel sparse recovery using convex relaxation," IEEE Trans. Inf. Theory, vol. 56, no. 1, pp. 505-519, Jan. 2010.

[12] D. Baron, M. Duarte, M. Waking, S. Sarvotham, and R. Baraniuk, "Distributed compressive sensing," 2005. Unpublished paper. Revised 2009. [Online]. Available: http://arxiv.org/pdf/0901.3403v1.pdf

[13] Y. Eldar and M. Mishali, "Robust recovery of signals from a structured union of subspaces," IEEE Trans. Inf. Theory, vol. 55, no. 11, pp. 53025316, Nov. 2009.

[14] S. Tebaldini, "Single and multipolarimetric SAR tomography of forested areas: A parametric approach," IEEE Trans. Geosci. Remote Sens., vol. 48, no. 5, pp. 2375-2387, May. 2010.

[15] J. Hagberg, L. Ulander, and J. Askne, "Repeat-pass SAR interferometry over forested terrain," IEEE Trans. Geosci. Remote Sens., vol. 33, no. 2, pp. 331-340, Mar. 1995.

[16] F. Bijma, J. de Munck, and R. Heethaar, "The spatiotemporal MEG covariance matrix modeled as a sum of Kronecker products," NeuroImage, vol. 27, no. 2, pp. 402-415, Aug. 2005.

[17] J. Mercer, "Functions of positive and negative type, and their connection with the theory of integral equations," in Proc. Roy. Soc. A, Math., Phys. Eng. Sci., 1909, vol. 83, pp. 69-70.

[18] M. Duarte and R. Baraniuk, "Kronecker compressive sensing," IEEE Trans. Image Process., vol. 21, no. 2, pp. 494-504, Feb. 2012.

[19] D. Donoho and J. Tanner, "Counting the faces of randomly-projected hypercubes and orthants, with applications," Discrete Comput. Geom., vol. 43, no. 3, pp. 522-541, Apr. 2010.

[20] M. Grant and S. Boyd, CVX: MATLAB software for disciplined convex programming, CVX Res., Austin, TX. [Online]. Available: http://cvxr. $\mathrm{com} / \mathrm{cvx}$ 